\section{ORIGINAL RESEARCH}

\author{
U.K. Udayasankar \\ K. Braithwaite \\ M. Arvaniti \\ D. Tudorascu \\ W.C. Small \\ S. Little \\ S. Palasis
}

\title{
Low-Dose Nonenhanced Head CT Protocol for Follow-Up Evaluation of Children with Ventriculoperitoneal Shunt: Reduction of Radiation and Effect on Image Quality
}

\begin{abstract}
BACKGROUND AND PURPOSE: Children with a shunt for hydrocephalus often undergo multiple follow-up head CT scans, increasing the risk for long-term effects of ionizing radiation. The purpose of our study was to evaluate if an unenhanced low-dose head CT could consistently provide acceptable image quality and diagnostic information.
\end{abstract}

\begin{abstract}
MATERIALS AND METHODS: Ninety-two children (mean age, 9 years; range, 8 months to 21 years; 45 boys and 47 girls) with a shunt for hydrocephalus and no clinical evidence of shunt malfunction who were referred for a follow-up nonenhanced head CT were included in the study. All studies were performed on a 4-section multidetector CT. Two CT studies were selected retrospectively for each patient, 1 performed at standard dose $(220 \mathrm{~mA})$ and 1 at low dose $(80 \mathrm{mAs})$. Two radiologists independently evaluated and graded both standard-dose and low-dose studies for various image quality parameters. Attenuation and noise levels were measured, and gray-white differentiation and contrast-to-noise ratio (CNR) were calculated.
\end{abstract}

RESULTS: Low-dose CT resulted in 63\% mean dose reduction. All low-dose CT scans were diagnostically acceptable. Image quality parameters were significantly lower at low dose $(P=.0001)$ except for the parameters for streak artifacts $(P=.46)$ and need for further imaging $(P=.47)$, which were higher. Mean noise levels were significantly higher $(P=.001)$ in low-dose studies, whereas CNR was significantly higher in standard dose CT $(P=.001)$. A moderate to perfect agreement was noted between the 2 readers with regard to image quality assessment $(65 \%-99 \%)$.

CONCLUSION: Low-dose nonenhanced head CT consistently provides diagnostically acceptable images with relevant diagnostic information in children with VP shunts resulting in substantial dose savings.
A ny imbalance in the production or resorption of CSF results in hydrocephalus. Surgical diversion of CSF via a ventriculoperitoneal (VP) shunt is the preferred method of treatment in most cases. More than 125,000 VP shunting procedures are performed every year in the United States. ${ }^{1}$ Radiologic evaluation is an essential component of pretreatment evaluation and postprocedural follow-up. Imaging studies are often used to assess the integrity of the shunt system, assess change in ventricular size, and identify any shunt-related complications. ${ }^{2} \mathrm{CT}$ is often the preferred technique because of its wide availability, ease of use, and brief imaging time. However, the foremost concern with increasing use of repeated CT studies in such patients is the associated dose of ionizing radiation and consequent potential risk of developing cancer later in life. ${ }^{3,4}$ Multiple factors including increased sensitivity of growing tissues to ionizing radiation, small cross-sectional area in children, and long latent period of oncogenic effects of ionizing radiation contribute to a more pronounced effect of ionizing radiation in children than in adults. ${ }^{5,6}$ Steps to minimize the risk for potential long-term complications of ionizing ra-

Received September 5, 2007; accepted after revision October 24

From Emory University Hospital (U.K.U, D.T., W.C.S.), Atlanta, Ga. and Children's Healthcare of Atlanta at Scottish Rite (K.B., M.A., S.L., S.P.), Atlanta, Ga.

Please address correspondence to Susan Palasis, MD, Department of Radiology, Children's Healthcare of Atlanta at Scottish Rite, 1001 Johnson Ferry Rd NE, Atlanta, GA 30342; e-mail: susan.palasis@choa.org

$\equiv$ indicates an article with supplemental on-line tables.

DOI 10.3174/ajnr.A0923 diation are therefore particularly relevant in children undergoing multiple follow-up CT scans. An arbitrary reduction in ionizing radiation may result in significant deterioration of image quality and may render CT studies clinically unacceptable. Low-dose CT protocols should attempt to balance image quality with radiation dose savings. Previous studies have shown the usefulness of low-dose CT protocols in the evaluation of pathologic processes throughout the body. ${ }^{7-12}$ The purpose of our study was to evaluate if a low-dose follow-up head CT study could provide relevant information in children with a VP shunt for hydrocephalus while maintaining acceptable image quality.

\section{Materials and Methods}

Our institutional review board approved this study, with waiver of informed consent. All CT examinations included in this study were performed as standard of care, and the results were retrospectively reviewed. The study protocol was in compliance with the Health Insurance Portability and Accountability Act.

\section{Patient Group}

Ninety-two consecutive subjects who underwent noncontrast head CT studies at our institution from April 2004 to August 2006 were included in this study. All subjects had VP shunting surgery performed to relieve intracranial pressure from hydrocephalus. The patients were referred to our department for routine follow-up CT to confirm absence of any shunt complications because these may not be clinically apparent in children with developmental delay. We incorporated low-dose CT scans in our routine scanning protocol in this 
specific patient population in an attempt to limit radiation dose yet retain pertinent diagnostic information. All subjects included in our study had 1 or more standard-dose $(220 \mathrm{~mA})$ unenhanced head CT scans as part of follow-up, before our present low-dose study. None of the patients in our study exhibited obvious clinical signs or symptoms of shunt malfunction. When such symptoms were present, those subjects were not included in our study, and a standard-dose head CT scan was performed.

\section{Scan Protocol}

All CT studies were performed on a 4-channel multidetector row CT (MDCT) scanner (LightSpeed QXi; GE Healthcare, Milwaukee, Wis). We performed a conventional standard-dose scan using the following parameters: tube current of $220 \mathrm{~mA}$; 1-second gantry rotation time (tube current-time product of $220 \mathrm{mAs}$ ), $120 \mathrm{kVp}$; and $10-\mathrm{mm} \mathrm{sec-}$ tion thickness at $10-\mathrm{mm}$ intersection distance. Scan parameters for low-dose CT studies remained the same except for tube current. A lower tube current of $80 \mathrm{~mA}$ was used for low-dose CT studies. Lowdose studies were performed in subjects who had a previous normal dose unenhanced CT.

\section{CT Radiation Dose}

The weighted average CT dose index (CTDIw) of a single CT section at $220 \mathrm{mAs}$ and $80 \mathrm{mAs}$ was calculated. The dose length products (DLP) were calculated from CTDIw and number of CT images. A constant region-specific normalized effective dose value was used $\left(0.0023 \mathrm{mSv} \mathrm{mGy}^{-1} \mathrm{~cm}^{-1}\right)$ to estimate effective doses from DLP. ${ }^{13}$

\section{Image Interpretation}

A total of 184 head CT scans (average number of images per scan, 16) were independently and retrospectively evaluated by 2 board-certified subspecialty radiologists on a digital PACS workstation (Centricity; GE Healthcare). The scans were randomized so that both readers initially evaluated either a high-dose or a low-dose study. The second study of each subject was evaluated after an interval of at least 2 weeks to eliminate potential recall bias. In addition, both readers were blinded to patient information and scan parameters. The only information available to both readers was that all subjects had VP shunting surgery for hydrocephalus. After statistical analysis, any significant differences in image quality parameters evaluated by the 2 readers were reevaluated by both readers to reach a consensus opinion.

\section{Image Quality Evaluation}

Both readers graded image quality parameters including noise, diagnostic acceptability (both on a 5 -point scale: 1 , unacceptable; 2 , suboptimal; 3 , average; 4, good; and 5, excellent), gray-white differentiation (scale of 3: 1, unacceptable; 2, suboptimal; 3, acceptable), sharpness of subarachnoid space margins (scale of 3: 1 , obscured and unacceptable; 2, suboptimal; 3, well-defined), visualization of posterior fossa structures (scale of 3: 1 , poor and unacceptable; 2 , suboptimal; 3 good), streak artifacts (scale of 3: 1, present and affecting diagnosis; 2, present but not affecting diagnosis (suboptimal); 3, absent), and need for further imaging (scale of 3: 1, definitely indicated; 2 , possibly indicated (suboptimal); 3 , not indicated). Image noise and diagnostic acceptability were considered acceptable when they were graded as 2 and above. Interpreting radiologists performed initial review of all images at preselected standardized window level and width. Diagnostic acceptability was graded as acceptable when the sharpness of ventricular outlines, tissue contrast, and shunt visualization were satisfactory; unacceptable when these image characteristics were unsatisfactory; and excellent when these imaging characteristics were equivalent to a high image quality standard-dose unenhanced head CT. Additional imaging studies were requested when imaging characteristics were unsatisfactory or when a lesion detected on CT studies needed further characterization with use of higher-dose, contrast-enhanced CT, or MR imaging. Streak artifacts were considered significant only when they were not produced by external factors including metallic artifacts.

\section{Quantitative Parameters of Image Quality}

One of the authors (U.U.) experienced in quantitative analysis of CT examinations performed the quantitative portion of the image analysis. A minimum of 4 regions of interest (ROIs) of the same size (4 $\mathrm{mm}^{2}$ ) were placed in identical locations for each pair of images in each patient, and attenuation coefficients were measured. The ROIs were placed in gray matter (GM) and white matter (WM) in selected supratentorial (centrum semiovale), and infratentorial (cerebellar parenchyma) regions, with particular attention paid to avoid inclusion of adjacent nonparenchymal structures (sulci, cisterns, and blood vessels) in the ROI to avoid partial volume effects. Density values (Hounsfield Units [HU]) for gray and white matter were averaged in standard-dose and low-dose scans for comparison and statistical evaluation. Noise was measured as SD in HU within the ROI selected. We calculated GM conspicuity and contrast-to-noise ratio (CNR) using the standard equations $\left({ }^{14,15}\right)$ :

$\mathrm{GM}$ conspicuity $=($ mean GM HU - mean WM HU)/mean WM

$$
\begin{aligned}
& \mathrm{HU} \mathrm{CNR}=(\text { mean GM HU }- \text { mean WM HU }) /\left[\left(\mathrm{SD}^{\star} \mathrm{GM} \mathrm{HU}\right)^{2}\right. \\
& \left.+(\mathrm{SD} \mathrm{WM} \mathrm{HU})^{2}\right]^{1 / 2} \\
& \quad{ }^{\mathrm{SD}} \text { is the standard deviation of } \mathrm{HU}
\end{aligned}
$$

\section{Statistical Evaluation}

We performed statistical evaluation of the data using commercially available statistical software (SAS Institute, Cary, NC). The Hotelling $\mathrm{T}$-square statistic was used to test the mean differences for several variables describing different measurements of image quality acquired by the use of 2 different radiation doses in CT. The Hotelling $\mathrm{T}$-square statistic is a generalization of the Student $t$ statistic that is used in multivariate hypothesis testing to evaluate the differences between the mean values of 2 groups. The null hypothesis is that the vector of means of several variables does not differ between 2 groups. We used multivariate analysis of variance to test for differences in radiation dose for each of the individual variables. In the case of 2 groups, all of the statistics are equivalent, and the test reduces to Hotelling T-square. We used the standard Student $t$ test to analyze quantitative parameters. We determined the degree of interobserver concordance by calculating the percentage of agreement between both readers. A $P$ value less than .05 was considered statistically significant.

\section{Results}

There were 92 subjects included in our study with a mean age of 9 years (range, $8-21$ years; SD, 3.4 years). There were 45 male and 47 female subjects in our study.

\section{CT Radiation Dose}

The weighted average CTDIw of a single CT section at $80 \mathrm{mAs}$ and $220 \mathrm{mAs}$ were $15.5 \mathrm{mGy}$ and $43.4 \mathrm{mGy}$, respectively. Lowdose CT scans performed at $80 \mathrm{mAs}$ (DLP, $252.8 \mathrm{mGyCm}$; 

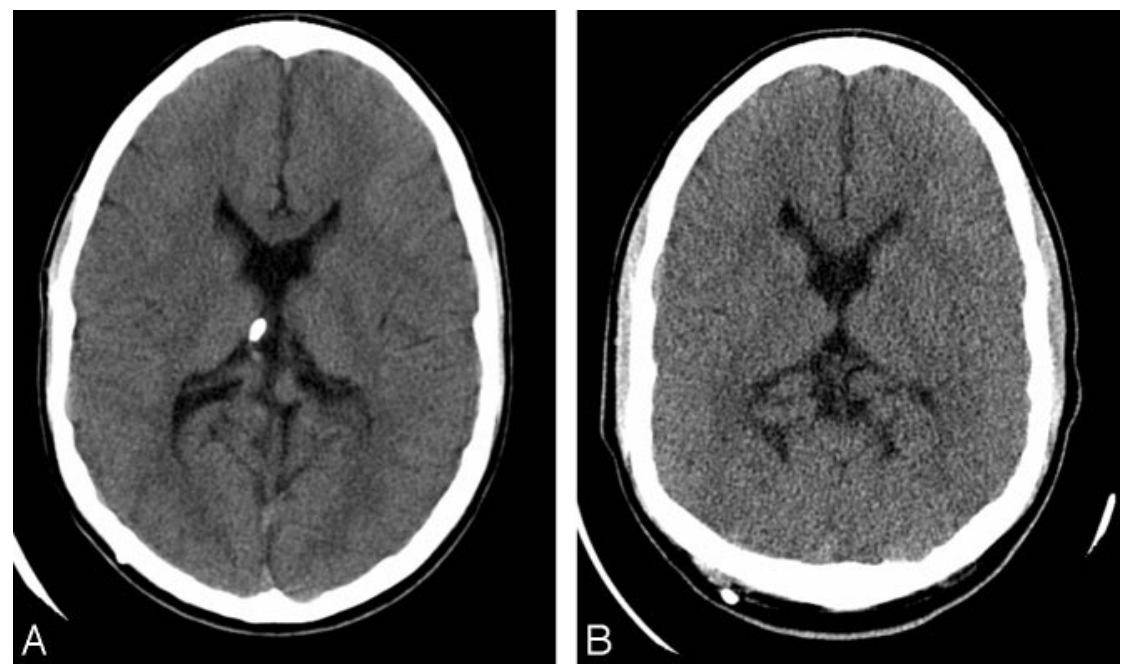

Fig 1. $A, B$, Diagnostic acceptability of low-dose CT studies. Standard-dose $(A)$ and low-dose $(B)$ head CT studies were obtained in a 16-year-old male adolescent at an interval of 8 months and were rated as good (grade 4) by both readers.

at $80 \mathrm{mAs}(0.72 \pm 0.04)$ was approximately $60 \%$ less than that of scans at 220 mAs $(1.84 \pm 0.11)$.

\section{Discussion}

The risk of developing ionizing radiationinduced cancer is higher in the pediatric age group compared with adults. The combination of higher dose of ionizing radiation and increased lifetime risk for ionizing radiation-induced carcinogenesis in children results in significantly increased risks for lifetime cancer mortality compared with

effective dose, $0.58 \mathrm{mSv}$ ) resulted in a reduction of $63.4 \%$ in effective radiation dose compared with scans at $220 \mathrm{mAs}$ (DLP, $695.1 \mathrm{mGyCm}$; effective dose, $1.6 \mathrm{mSv}$ ).

\section{Image Quality and Adequacy for Diagnosis}

None of the standard-dose and low-dose head CT studies were rated diagnostically unacceptable (score of 1 ) by either reader (Fig 1). However, low-dose CT studies produced more suboptimal images (15 for reader 1 and 6 for reader 2) compared with standard-dose CT examinations ( 2 for reader 1 and 5 for reader 2 ) as per ratings by the 2 respective readers; this difference was significant for both readers $(P=.0002$ and $P=.0003$ for readers 1 and 2, respectively). Summaries of assessment of image quality by each reader are provided in On-line Tables 1 and 2.

Mode (most frequently occurring score of image quality assessment) is considered a better indicator of frequency distribution than mean or median in ordinal data such as image quality scoring in our study. The modal score of image quality evaluation by both readers for all image parameters was 3 (corresponding to acceptable diagnostic quality). Significant differences were detected between the 2 radiation doses for all image quality variables except for streak artifacts $(P=.4695$ and $P=.5680)$ and need for further imaging $(P=.4724$ and $P$ $=.5723$ ) for readers 1 and 2 , respectively. Other image quality parameters for standard-dose CT were significantly better than those of low-dose CT scan $(P<.05)$. There was moderate to excellent interobserver agreement in the assessment of various image quality parameters $(65 \%-99 \%)$

\section{Quantitative Evaluation}

Analysis of attenuation characteristics of GM and WM did not reveal any significant difference in $\mathrm{HU}$ values between the studies performed at $220 \mathrm{mAs}(33.7$ and $25.9 \mathrm{HU})$ and $80 \mathrm{~mA}$ (34.7 and 25.7 HU) $(P=.10$ and $P=.80)$ (Fig 2). However, mean GM conspicuity was significantly different for the 220 and $80 \mathrm{~mA}$ studies $(0.33 \pm 0.11,0.31 \pm 0.09 ; P=.003)$. As expected, the mean GM and WM noises were significantly higher $(P=.0001$ and $P=.001)$ with scans at $80 \mathrm{mAs}$ (3.7 and $3.6 \mathrm{HU})$ compared with scans at $220 \mathrm{~mA}$ (2.2 and $2.0 \mathrm{HU})$. CNR was significantly higher $(P<.001)$ for higher-dose studies than that of low-dose CT studies; the mean CNR for studies adults. ${ }^{10,16}$ Although pediatric CT examinations still constitute a small fraction of the overall number of CT examinations performed, recent advances in CT technology have exponentially increased the number of pediatric CT examinations. ${ }^{11}$ Current CT scanners offer faster scanning techniques that result in reduced need for sedation. ${ }^{17,18}$ These scanners also facilitate more practical examinations in younger, sicker, and uncooperative children, thus contributing to an increasing number of CT studies and hence the potential for the cumulative effects of ionizing radiation. The burden of increased ionizing radiation is particularly important in children undergoing multiple follow-up scans for chronic or complex disorders (hydrocephalus, tumors, trauma, and vascular disease) and to assess the impact of management strategies. In an ideal setting, CT examinations that are properly performed in children should expose children to much lower doses of ionizing radiation than for the same procedure in an adult. Our study shows the usefulness of a low-dose head CT protocol in the evaluation of patients after placement of a VP shunt.

In pediatric neuroradiology, it is not uncommon for children with VP shunts to undergo multiple follow-up CT scans to assess the patency of the shunt and to assess any complications. Although initial scans may focus on finding abnormal pathologies, subsequent scans are usually oriented toward evaluation of the shunt, determination of stability of ventricular size, and identification of related complications. We evaluated the utility of a lower-dose head CT scan protocol using a lower tube current-time product for follow-up studies. Our study shows that diagnostically acceptable image quality could be maintained at $80 \mathrm{mAs}$ with significant reduction in ionizing radiation. The low-dose CT protocol $(80 \mathrm{mAs})$ in our study resulted in a savings of ionizing radiation of approximately $63 \%$ compared with standard-dose studies (220 mAs). As shown in previous studies, the overall risk for ionizing radiation-induced cancer is dependent on tube current-time product (in mA-seconds) and frequency of CT scans in children. ${ }^{16,19}$ Low-dose CT studies resulted in an effective radiation dose of $0.58 \mathrm{mSv}$, which is comparable with diagnostic radiation reference levels for skull anteroposterior (0.54 $\mathrm{mSv})$ and lateral $(0.35 \mathrm{mSv}) \mathrm{x}$-rays. ${ }^{20}$ Recent technologic advances in MDCT scanners combined with ultra-low-dose protocols may result in radiation doses comparable with standard 

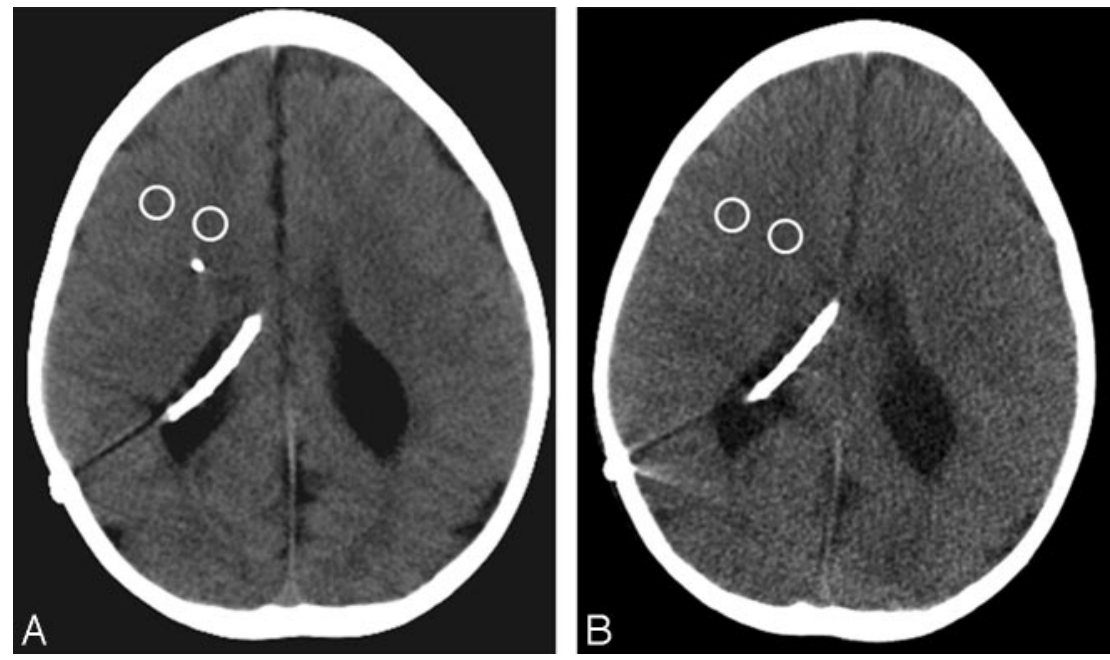

skull x-rays. However, additional studies are warranted to maximize the effect of these protocols and balance the image quality with dose savings.

Although low-dose studies resulted in more images with suboptimal image quality compared with standard-dose CT scans, both readers rated all images as diagnostically acceptable. Statistical evaluation of image quality parameters showed significantly better scores for most standard-dose scans. It is more important that both readers did not recommend further imaging in any of the cases despite the differences in image quality. Results of our study conform to a previous one performed on adult patients by Mullins et $\mathrm{al}^{15}$ that compared the image quality of head CT scans at $90 \mathrm{mAs}$ with those at 170 $\mathrm{mAs}$ and rated all low-dose images as diagnostically acceptable despite the moderately increased noise levels. Results of visual image quality ratings, CNR, and noise levels obtained in our study were comparable with those in the study by Mullins et al. $^{15}$

Various technical modifications in CT scanners can result in substantial savings of radiation doses. Altering tube current is a commonly adopted strategy because ionizing radiation has a direct linear relationship with $\mathrm{mA} \cdot{ }^{21}$ However, alterations in tube potential $(\mathrm{kV})$, pitch (in helical scanners), and section thickness can also contribute to dose savings. Bow-tie filtration and automatic tube current modulation are techniques in MDCT scanners used to reduce the effective ionizing radiation received to the organ being scanned. Substantial dose savings have been reported with routine use of these techniques on CT of the abdomen, chest, and neck. ${ }^{22-24}$ Significant dose savings have also been reported in MDCT of the extremities in children with automatic tube current modulation techniques. ${ }^{25}$ Cohnen et $\mathrm{al}^{26}$ assessed the changes in image quality in reduced doses of head CT scans by lowering both $\mathrm{mA}$ and $\mathrm{kV}$ in cadavers and achieved a dose reduction of up to $40 \%$ without loss of diagnostic image quality. However, assessing the effect of low $\mathrm{kVp}$ on image quality was not within the purview of our study, but low $\mathrm{kVp}$ may also result in significant dose savings without altering image quality, and we believe that this topic may particularly benefit from additional investigations.

The most important limiting factor of studies at low $\mathrm{mA}$ is the associated high noise. Low-dose CT scans in our study cols in other regions of the body. Image noise at a setting of low $\mathrm{mAs}$ may seem exaggerated if viewed at the same window level settings compared with the standard protocol images. This shortcoming could be overcome by increasing the window width by the same factor as the noise increase. This would produce a similar visual appearance in the gray-scale image as with the lower noise at the settings at higher $\mathrm{mA}$. Noise reduction filters can also help reduce image noise and may represent another tool to render lower-dose scans acceptable for clinical use. These filters, when applied to low-dose CT studies, work on the principle of filtering pixels representing nonstructural data from pixels representing structures of interest in images. $^{27,28}$

There were a few limitations to our study. One was that the standard-dose and low-dose CT scans in our study were not concurrently acquired. Simultaneous acquisition of both scans would have enabled more accurate comparison of image quality parameters. Standard-dose CT scan would also have served as a reference standard against which the diagnostic quality and imaging findings of low-dose scans could easily have been assessed. Because our study group consisted mostly of young children and therefore a consequent higher risk for ionizing radiation, we did not subject these patients to additional scanning. Also, because the purpose of our study was to assess the relative usefulness of low-dose studies in follow-up of positioning of the shunt and associated complications, a direct comparison with high dose scan was outside the scope of our study. Another limitation of our study was the ability of both readers to recognize low-dose studies from standarddose scans during the retrospective review. Although both readers were blinded to the technical details of each image, higher noise levels on images at $80 \mathrm{mAs}$ made them stand out and could have affected the overall scoring pattern of image quality. CT scanning with reduced $\mathrm{mAs}$ may limit the ability to detect low-contrast ${ }^{29}$ or tiny lesions, especially those in the posterior fossa. Evaluation of the effect of low-dose head CT scan in the detection of tiny lesions was also not part of our study. In addition, we did not evaluate alternate techniques that do not involve radiation to evaluate these patients. Cranial sonography in the first few months of life and faster MR imaging techniques could serve as practical alternatives to CT. 


\section{Conclusion}

Increased doses of ionizing radiation are a serious concern in children with a shunt for hydrocephalus requiring multiple follow-up head CT examinations. A low-dose head CT protocol for such patients resulted in diagnostically acceptable images with reduction in radiation dose of approximately $63 \%$. Although image quality ratings of a low-dose-study were lower than those of standard-dose scans, the quality of images was adequate for the assessment of ventricular volume and shunt patency. Our study shows that a low-dose head CT is an acceptable technique to replace standard-dose CT in pediatric patients undergoing follow-up scans of a shunt for hydrocephalus.

\section{References}

1. Bondurant CP, Jimenez DF. Epidemiology of cerebrospinal fluid shunting. Pediatr Neurosurg 1995;23:254-58; discussion 259

2. Browd SR, Ragel BT, Gottfried ON, et al. Failure of cerebrospinal fluid shunts: part I: Obstruction and mechanical failure. Pediatr Neurol 2006;34:83-92

3. Mills DM, Tsai S, Meyer DR, et al. Pediatric ophthalmic computed tomographic scanning and associated cancer risk. Am J Ophthalmol 2006;142:1046-53

4. Rice HE, Frush DP, Farmer D, et al. Review of radiation risks from computed tomography: essentials for the pediatric surgeon. J Pediatr Surg 2007;42:603-07

5. Hall EJ. Lessons we have learned from our children: cancer risks from diagnostic radiology. Pediatr Radiol 2002;32:700-06

6. Huda W, Atherton JV, Ware DE, et al. An approach for the estimation of effective radiation dose at CT in pediatric patients. Radiology 1997;203:417-22

7. Donnelly LF, Emery KH, Brody AS, et al. Minimizing radiation dose for pediatric body applications of single-detector helical CT: strategies at a large children's hospital. AJR Am J Roentgenol 2001;176:303-06

8. Lucaya J, Piqueras J, Garcia-Pena P, et al. Low-dose high-resolution CT of the chest in children and young adults: dose, cooperation, artifact incidence, and image quality. AJR Am J Roentgenol 2000;175:985-92

9. Mulkens TH, Broers C, Fieuws S, et al. Comparison of effective doses for lowdose MDCT and radiographic examination of sinuses in children. AJR Am J Roentgenol 2005;184:1611-18

10. Robinson AE, Hill EP, Harpen MD. Radiation dose reduction in pediatric CT. Pediatr Radiol 1986;16:53-54

11. Vock P. CT dose reduction in children [published erratum appears in Eur Radiol 2005;15:2383-84]. Eur Radiol 2005;15:2330-40
12. Rybka K, Staniszewska AM, Biegański T. Low-dose protocol for head CT in monitoring hydrocephalus in children. Med Sci Monit 2007;13:147-51

13. European Study Group of Physicists. European Guidelines on Quality Criteria for Computed Tomography EUR 16262 EN; 1999. Available at: http://www. drs.dk/guidelines/ct/quality/mainindex.htm. Accessed on 01/02/2008.

14. Lev MH, Kulke SF, Sorensen AG, et al. Contrast-to-noise ratio in functional MRI of relative cerebral blood volume with sprodiamide injection. J Magn Reson Imaging 1997;7:523-27

15. Mullins ME, Lev MH, Bove $\mathrm{P}$, et al. Comparison of image quality between conventional and low-dose nonenhanced head CT. AJNR Am J Neuroradiol 2004;25:533-38

16. Brenner D, Elliston C, Hall E, et al. Estimated risks of radiation-induced fatal cancer from pediatric CT. AJR Am J Roentgenol 2001;176:289-96

17. Frush DP, Donnelly LF. Helical CT in children: technical considerations and body applications. Radiology 1998;209:37-48

18. White KS. Invited article: helical/spiral CT scanning: a pediatric radiology perspective. Pediatr Radiol 1996;26:5-14

19. Karabulut N, Ariyürek M. Low dose CT: practices and strategies of radiologists in university hospitals. Diagn Interv Radiol 2006;12:3-8

20. Aroua A, Besançon A, Buchillier-Decka I, et al. Adult reference levels in diagnostic and interventional radiology for temporary use in Switzerland. Radiat Prot Dosimetry 2004;111:289-95

21. Kalra MK, Prasad S, Saini S, et al. Clinical comparison of standard-dose and $50 \%$ reduced-dose abdominal CT: effect on image quality [published erratum appears in AJR Am J Roentgenol 2002;179:1645]. AJR Am J Roentgenol 2002;179:1101-06

22. Kalra MK, Rizzo S, Maher MM, et al. Chest CT performed with $\mathbf{z}$-axis modulation: scanning protocol and radiation dose. Radiology 2005;237:303-08

23. Namasivayam S, Kalra MK, Pottala KM, et al. Optimization of z-axis automatic exposure control for multidetector row CT evaluation of neck and comparison with fixed tube current technique for image quality and radiation dose. AJNR Am J Neuroradiol 2006;27:2221-25

24. Rizzo SM, Kalra MK, Schmidt B. Automatic exposure control techniques for individual dose adaptation. Radiology 2005;235:335-36; author reply 336

25. Salamipour H, Jimenez RM, Brec SL, et al. Multidetector row CT in pediatric musculoskeletal imaging. Pediatr Radiol 2005;35:555-64

26. Cohnen M, Fischer H, Hamacher J, et al. CT of the head by use of reduced current and kilovoltage: relationship between image quality and dose reduction. AJNR Am J Neuroradiol 2000;21:1654-60

27. Mastora I, Remy-Jardin M, Delannoy V, et al. Multi-detector row spiral CT angiography of the thoracic outlet: dose reduction with anatomically adapted online tube current modulation and preset dose savings. Radiology 2004;230:116-24

28. Kachelriess M, Watzke O, Kalender WA. Generalized multi-dimensional adaptive filtering for conventional and spiral single-slice, multi-slice, and conebeam CT. Med Phys 2001;28:475-90

29. McNitt-Gray MF. AAPM/RSNA Physics Tutorial for Residents: Topics in CT. Radiation dose in CT. Radiographics 2002;22:1541-53 